\title{
Infliximab treatment in a patient with rheumatoid arthritis and pulmonary fibrosis
}

\section{To the Editors:}

Infliximab is a chimeric human-mouse anti-tumour necrosis factor- $\alpha$ (anti-TNF- $\alpha$ ) immunoglobulin $G$ monoclonal antibody that binds the soluble and transmembrane forms of TNF- $\alpha$. Its efficacy in the treatment of chronic inflammatory diseases, such as rheumatoid arthritis (RA) and Crohn's disease, is well documented $[1,2]$, and recent reports indicate a possible use in the treatment of psoriasis, giant cell arteritis, spondyloarthropaties and sarcoidosis [3-6].

As conventional treatments of idiopathic pulmonary fibrosis and lung fibrosis associated to RA are not effective, it is interesting to refer the efficacy of $T N F-\alpha$ inhibitor therapy in a patient affected by interstitial lung disease and RA (diagnosis performed according to the American College of Rheumatology classification criteria [7]). The patient was a 70-yr-old female, nonsmoker, refractory to azathyoprine and steroids. As recommended for RA [2], Remicade $3 \mathrm{mg} \cdot \mathrm{kg}^{-1}$ was administered at time 0 , after $2-6$ weeks, then every 8 weeks, and it was associated with methotrexate $\left(10 \mathrm{mg} \cdot \mathrm{week}^{-1}\right)$ and folic acid supplementation. The treatment was well tolerated and after the second infusion, joint symptoms improved dramatically, with a substantial reduction in morning stiffness, inflammatory index (C-reactive protein and erythrocyte sedimentation rate) and number of tender joints. During the treatment arterial oxygen tension and carbon dioxide arterial tension remained stable in the normal range. High-resolution computed tomography scan of the chest, that showed bibasilar mantellar fibrosis with honeycombing, was unchanged after 3, 6, 12 and 15 months of therapy.

Before starting Infliximab, lung function tests (LFT) showed a restrictive pattern with a progressive worsening of vital capacity (VC) and transfer factor of the lung for carbon monoxide $(T \mathrm{~L}, \mathrm{Co})$. After 15 months of Infliximab, there were increases of $17 \%$ in $T \mathrm{~L}, \mathrm{Co}(65 \%$ of theoretical value at time 0 and $82 \%$ after 15 months of Infliximab) and $11 \%$ in VC (73\% at time 0 and $84 \%$ after 15 months of therapy), and a stabilisation of forced expiratory volume in one second $(73 \%$ of the predicted value at time 0 and $76 \%$ after 15 months of therapy).

These results indicate that Infliximab may have positive effects in the treatment of rheumatoid arthritis associated with interstitial lung disease, as also reported by VASSALLO et al. [8], who treated a patient with rheumatoid arthritis and lung fibrosis for $1 \mathrm{yr}$ with anti-tumour necrosis factor- $\alpha$. These two reports suggest that Infliximab (with or without methotrexate [8]) may be a therapeutic option for the treatment of pulmonary fibrosis associated with rheumatoid arthritis.

\section{E. Bargagli*, M. Galeazzi ${ }^{\#}$, P. Rottoli*}

*Respiratory Diseases Section, and "Rheumatology Section, Dept of Clinical Medicine and Immunology, Siena University, Siena, Italy.

\section{References}

1. Legnan P, Kornbluth A. Newer therapies for inflammatory bowel disease. Curr Treat Options Gastroenterol 2004; 7: 161167.

2. Maini RN, Elliott MJ, Brennan FM. Monoclonal anti-TNF $\alpha$ antibody as a probe of pathogenesis and therapy of rheumatoid disease. Immunol Rev 1995; 144: 195-223.

3. Goedkoop AY, Kraan MC, Teunissen MB, et al. Early effects of TNF alpha blokade on skin and synovial tissue in patients with active psoriasis and psoriasis arthritis. Ann Rheum Dis 2004; 63: 769-773.

4. Andonopoulos AP, Meimaris N, Daoussis D, Bounas A, Giannopoulos G. Experience with Infliximab as monotherapy for giant cell arteritis. Ann Rheum Dis 2003; 62: 1116.

5. Labarca C, Massardo L, Garcia PI, Jacobelli S. Effectiveness of Infliximabin patients with inflammatory arthritis refractory to conventional treatment. Rev Med Chil 2003; 131: $1157-1164$.

6. Baughman RP, Lower E. Infliximab for refractory sarcoidosis. Sarcoidosis Vasc Diffuse Lung Dis 2001; 18: 70-74.

7. Saraux A, Bethelot JM, Chales G, et al. Ability of the American College of Rheumatology 1987 criteria to predict rheumatoid arthritis in patients with early arthritis and classification of these patients two years later. Arthritis Rheum 2001; 44: 2485-2491.

8. Vassallo R, Matteson E, Thomas CF. Clinical response of rheumatoid arthritis associated pulmonary fibrosis to tumor necrosis factor- $\alpha$ inhibition. Chest 2002; 122: 1093-1096.

\section{Nasal versus full face mask for noninvasive ventilation in chronic respiratory failure}

\section{To the Editor:}

I read with interest the study by WILLSON et al. [1] published recently in the European Respiratory Journal. The paper was focused on interfaces, which are one of the most important issues in treating patients with chronic respiratory failure. The study was set up to compare nasal mask (NM) with full face mask (FFM) ventilation, in terms of sleep quality, gas exchange and tolerability. In addition, the authors tried to investigate any differences in pressure settings between the 
two types of mask ventilation. Although the questions of this study are relevant, I believe that the design of the study was not adequate to answer these questions.

First, all patients were on NM for $>3$ months and one patient even for 70 months, whereas they trained only for 1 day on FFM. In our centre (Dept of Pulmonary Diseases/ Home mechanical ventilation, University Hospital Groningen, Groningen, The Netherlands), patients routinely need more time ( $\sim 7$ days) to adjust to their specific mask. As a result of this short time of training on FFM compared with a long-term adjustment on NM, it is not surprising that total sleep time and sleep efficiency were worse in the FFM group compared with the NM group. As a consequence of their longer experience with the NM mask, the patients also rated less leak and more comfort in the NM night compared with the FFM night. Secondly, no differences were found between pressure settings if NM was compared with FFM. However, this study will not detect any differences if pressure settings, optimalised during their review night, remained unchanged during the subsequent mask trials.

Due to the previously mentioned issues, I believe that the study by WILLSON et al. [1], with its present design, makes it difficult to draw strong conclusions. It would have been preferable to compare both mask ventilations in a parallel study of naïve patients in whom noninvasive ventilation was started. In this way, it would have been possible to compare the time it takes to adjust to either nasal mask or full face mask. If the patients were adjusted to the mask, it would be possible to determine differences in terms of sleep quality, tolerability and pressure needed to maintain an effective noninvasive ventilation.

\section{P. Wijkstra}

Dept of Pulmonary Diseases/Home mechanical ventilation, University Hospital Groningen, Groningen, The Netherlands.

\section{References}

1. Willson GN, Piper AJ, Norman M, et al. Nasal versus full face mask for noninvasive ventilation in chronic respiratory failure. Eur Respir J 2004; 23: 605-609.

DOI: $10.1183 / 09031936.04 .00054204$

\section{From the authors:}

We welcome the interest of P. Wijkstra in our paper [1]. We agree that the choice of an effective interface for noninvasive ventilation (NIV) is integral to the success of this therapy and would welcome future research in the area. We acknowledge that our study was not without methodological weaknesses, a number of which we mention in the discussion of our paper [1]. Whilst we would now consider a trial in naive patients, this was not the situation at the commencement of data collection for our study. At that time the safety and efficacy of the full face mask (FFM) for NIV during sleep was largely unknown. Our adverse experience with this interface led us to be cautious with regard to its efficacy during sleep, especially in subjects with neuromuscular disease. Indeed, this concern was borne out in the study with the observation of upper airway obstruction in one subject on FFM versus an absence of respiratory events on the nasal mask. We would still recommend careful attention to the degree of upper airway closure during sleep in subjects using a FFM.

It was with these considerations in mind that rather than choosing naïve subjects we chose a group of subjects who were receiving effective NIV therapy via a nasal mask (NM). In this sense we were well aware that the NM versus FFM comparison may be weighted toward the NM. Alternatively, the acute application of a more effective interface may be argued to have biased our study toward the FFM. The proposals of $\mathrm{P}$. Wijkstra in regard to the mask acclimatisation are well thought out and in line with many of our own clinical observations. We concur with P. Wijkstra that our study did not directly address the question of the difference in pressure requirements on the FFM. Our aim would have been more appropriately worded: To determine if the pressures titrated during NM ventilation were effective during FFM ventilation. We believe our study does show that the pressure settings titrated whilst wearing the NM will be adequate if the patient is changed to a FFM.

We would hope that the results of our study will be interpreted in light of the subjects studied and the methodology used. We would direct readers to the broader conclusions of our study that full face masks appeared to be as effective as nasal masks for the delivery of noninvasive ventilation.

\section{G.N. Willson, A.J. Piper, R.R. Grunstein}

Dept of Respiratory Medicine, Royal Prince Alfred Hospital, Camperdown, Sydney, New South Wales, Australia.

\section{References}

1. Willson GN, Piper AJ, Norman M, et al. Nasal versus full face mask for noninvasive ventilation in chronic respiratory failure. Eur Respir J 2004; 23: 605-609.

DOI: $10.1183 / 09031936.04 .00063904$

\section{Methacholine and macrolides}

\section{To the Editor:}

The paper by Kostadima et al. [1] provides a valuable insight into the effects of clarithromycin upon nonspecific airway hyperresponsiveness (AHR) to methacholine in asthmatics.

It is important to point out that AHR to methacholine is only very loosely related to the degree of underlying inflammation, whereas the use of an indirect bronchoconstrictor stimulus in the KOSTADIMA et al. [1] study would have provided more convincing evidence of anti-inflammatory activity [2]. Bronchoprovocation with indirect stimuli, such as adenosine monophosphate (AMP), is considered to be particularly relevant to real life situations, since cold air, exercise and allergens also act in a similar fashion, in terms of the release of inflammatory mediators from primed mast cells. Indeed, shifts in the AMP threshold are more closely related to underlying airway eosinophilic inflammation and associated with symptoms of atopic asthma than direct stimuli, such as methacholine [3, 4]. 